\title{
Office hysteroscopic cervical myomectomy
}

\author{
Bernardo Portugal Lasmar • Ricardo Bassil Lasmar • Claudia Pillar
}

Received: 8 October 2012 / Accepted: 29 November 2012 / Published online: 22 December 2012

(C) Springer-Verlag Berlin Heidelberg 2012

\section{Introduction}

Leiomyomas are the most common tumors of the uterus and the female pelvis, with an estimated prevalence of $40-50 \%$. The main symptom is transvaginal bleeding being responsible for high levels of hysterectomy worldwide [1]. Other complaints are dyspareunia, abdominopelvic discomfort, and lower abdominal pain. They are benign tumors composed mainly of smooth muscle cells associated with fibrous connective tissue in varying amounts. The clinical treatment can be accomplished with gestrinone, $\mathrm{GnRH}$ analogues, and danazol in premenopausal women seeking improvement in bleeding until menopause. GnRH analogue may be a therapeutic option for 3 to 6 months; when necessary for a longer period, it should be combined with gonadotropic hormones to minimize the effects of bone demineralization.

The surgical approach, myomectomy, is the definitive treatment of disease and may be done by laparotomy, laparoscopy, or hysteroscopy, depending on the number, size, and location of the nodules. In some cases, a hysterectomy may be indicated, particularly in women with no desire of future pregnancy. Most fibroids are situated in the uterine body, with a minority (less than $5 \%$ ) in the cervical canal $[2,3]$. The approach to the cervical myoma is complex, since it is closer to the ureters, bladder, rectum, and cervical vessels [3].

Hysteroscopic myomectomy is a complex surgery indicated in cases of submucosal fibroids. The main complications of the procedure are bleeding, uterine perforation, and overload. The procedure can be done as office hysteroscopic myomectomy using biopsy punch and scissors or bipolar electrodes, or as hospital hysteroscopic myomectomy with cervical dilation using mono or bipolar resectoscope.

B. P. Lasmar $(\varangle) \cdot$ R. B. Lasmar · C. Pillar

Department of Gynecology, Fluminense Federal University,

Rua Marques do Paraná 303,

Niteroi, Rio de Janeiro, Brazil

e-mail: bernardolasmar@gmail.com
In 2005, Lasmar et al. developed the STEPW classification $[4,5]$ which provides the degree of difficulty of hysteroscopic myomectomy by classifying the fibroids preoperatively. This classification includes intracavitary fibroids only; cervical fibroids are not contemplated. Hysteroscopic myomectomy may be performed by different techniques: direct mobilization, slicing, or both $[6,7]$. It can be used with $\mathrm{U}$ or $\mathrm{L}$ resectoscope handle, with or without energy, either monopolar or bipolar. In direct mobilization, the Collins electrode is used in shape of an " $\mathrm{L}$ " to dissect the endometrium around the fibroid. The "cold electrode" is used to mobilize the fibroid in all directions, doing the coagulation only of the vessels that are bleeding. After releasing the nodule from the uterine wall, it can be removed by grasping forceps. If the fibroid is too large, it can be sliced in several pieces using the Collins electrode [6].

In office hysteroscopic procedure, we prefer to use the direct mobilization technique as well with biopsy punch, sectioning fibrous beams of the myoma pseudocapsule as it is mobilized. Saline infusion is used as distension media. This technique is similar to that in the hospital setting and allows the resection of fibroid very close to the uterine safely and efficiently $[8,9]$.

The literature has few data on the management of cervical myomas, with most referrals regarding cervical myomectomy by laparoscopy route $[3,10]$. The approach to cervical fibroids by hysteroscopy is possible, mainly on those with little intramural component, and should be done cautiously due to the small wall thickness and short proximity to important structures of the pelvis [3]. The presence of large masses leads to a dilatation of the cervical canal, difficulting its distension and making the procedure even harder.

\section{Case presentation}

A 46-year-old woman attended the gynecology ambulatory of University Antonio Pedro Hospital, complaining of abnormal uterine bleeding. Her gynecologic history was two 
pregnancies with two vaginal deliveries. Her Pap smear results were normal. She had not undergone other surgeries. Ultrasound imaging, made 6 months before, showed an anteflexed uterus measuring $8.0 \times 4.9 \times 3.7 \mathrm{~cm}$, with a 10 $\mathrm{mm}$ hypoechoic and heterogenic mass at cervical topography. The uterine cavity was normal and with an endometrial thickness of $4 \mathrm{~mm}$.

An office hysteroscopy was indicated. The patient was counseled, and the hysteroscopy showed a $25-\mathrm{mm}$ cervical mass with regular base at medium cervical canal into the right anterior wall (Figs. 1 and 2). The uterine cavity showed a proliferative endometrium and no lesions (Fig. 3). The cervical lesion was extracted with a 5 Fr biopsy punch using mobilization myomectomy technique, cutting the myoma pseudocapsule with the same instrument. The lower cervical canal and the external uterine ostium were already dilatated by the myoma, making the distension of the canal difficult but allowing the removal of the fibroid in one piece. The procedure time was $35 \mathrm{~min}$, and the patient referred no pain. A 4.0-mm rigid $30^{\circ}$ hysteroscope with operating sheath and with a straight $5 \mathrm{Fr}$ working channel was used. Saline was used as the distension media.

The hystophatological study showed a $2.5 \times 1.0 \times 1.5 \mathrm{~cm}$ nodular fragment (Fig. 4) with brown areas, fibroelastic consistence, and containing small cystic areas. In conclusion, this was a myoma with adenomyosis.

\section{Discussion}

Cervical myomectomy can be performed abdominally (laparotomy or laparoscopy) or vaginally by hysteroscopy [10]. The route to be used depends on the size and location of fibroids, as well as the experience of the surgeon and staff. It is established that laparoscopic

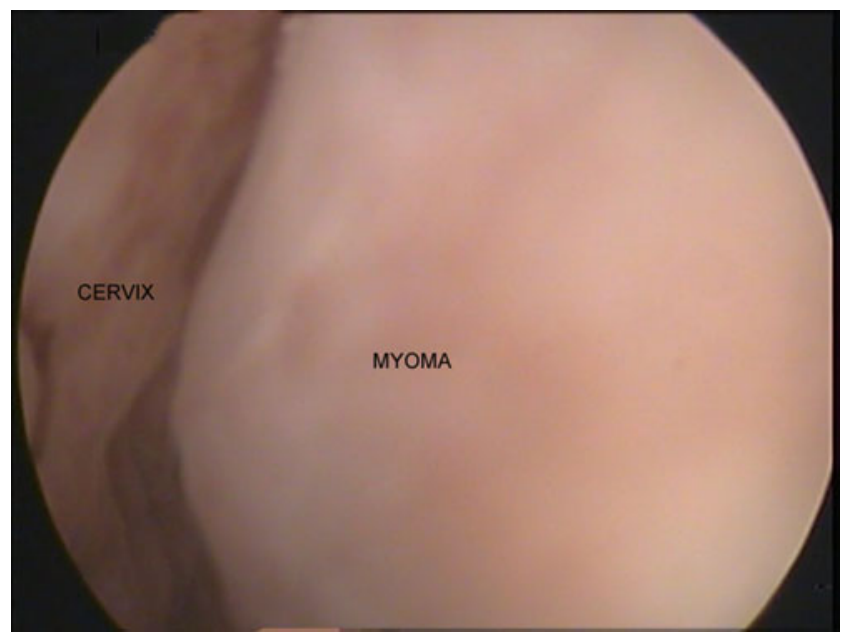

Fig. 1 Hysteroscopic image of a 25-mm cervical mass with regular base at medium cervical canal into the right anterior wall

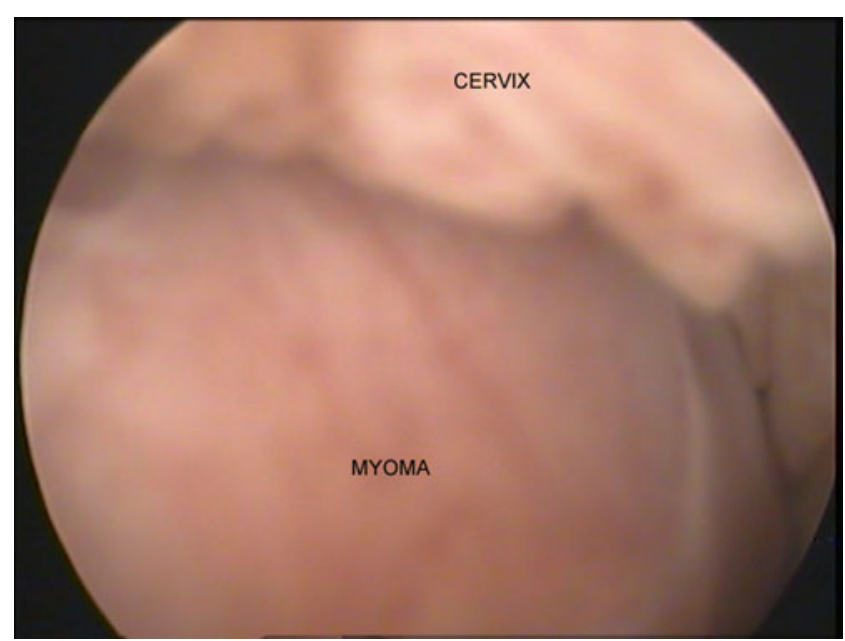

Fig. 2 A $2.5 \times 1.0 \times 1.5 \mathrm{~cm}$ nodular fragment with brown areas, fibroelastic consistence, and containing small cystic areas

route should be performed for large cervical fibroids with significant intramural component and hysteroscopic myomectomy for those restricted to the cervical canal. The total hysterectomy is an option; however, it prevents future pregnancy and is a procedure with greater morbidity and mortality. The option for supracervical hysterectomy obviously does not fit in the case of cervical fibroids, and if it is done, it does not prevent the formation of cervical fibroids in the remaining cervix [11].

Besides the proximity of important structures, the cervical canal is narrow, and the presence of a fibroid in this region makes it very difficult to access and approach the lesion. Moreover, the closer the fibroid to the external orifice, the greater the difficulty is the distension of the canal due to loss of distension media, making the procedure even more difficult.

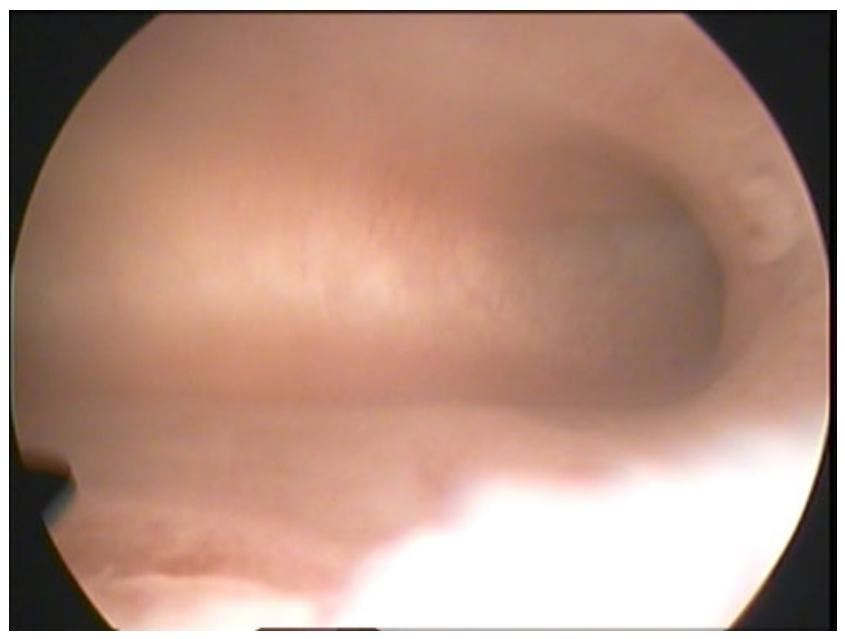

Fig. 3 The uterine cavity showed a proliferative endometrium and no lesions 


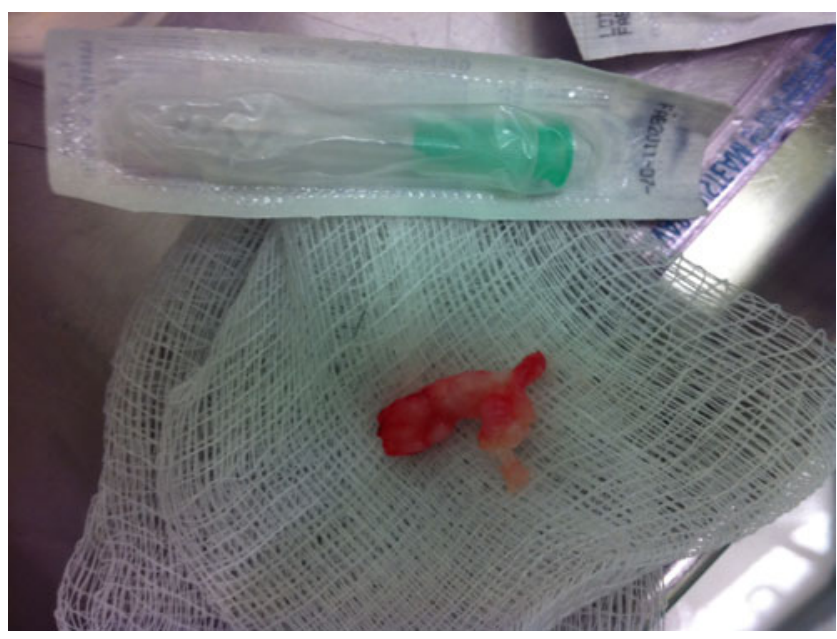

Fig. 4 Myoma after hysteroscopic removal

Office cervical myomectomy is a safe procedure and widely performed in our institution. The patient must be informed about the risks of the procedure, and anesthesia should be indicated if the patient refers pain or complains of too much discomfort, although it is not frequent. The patient must consent and have a good interaction with the team. Fibroids level 0 and some level 1 can be removed without anesthesia in the same procedure of hysteroscopy. The myomectomy technique is the direct mobilization of the fibroid [5], uses no energy of any kind, allows the use of smaller diameter material, and causes less discomfort for the patient. It is also possible to use bipolar energy with small diameter instrument, allowing office hysteroscopic myomectomy to be performed with less discomfort and securely [12].

\section{Conclusion}

Office cervical myomectomy is a safe and well-tolerated procedure for both cervical and intracavitary myomas, low cost for the patient, and without risk of thermal accident, using direct mobilization technique and biopsy punch instrument.
Conflict of interest The authors report no conflicts of interest. The authors alone are responsible for the content and writing of the paper.

\section{References}

1. Thurkow AL, Admiraal CF, Emanuel MH, van Kesteren PJM, Veersema S (2008) Submucous myomas: diagnosis and therapy. Gynecol Surg 5(2):93-102

2. Patel P, Banker M, Munshi S, Bhalla A (2011) Handling cervical myomas. J Gynecol Endosc Surg 2(1):30-32

3. Matsuoka S, Kikuchi I, Kitade M, Kumakiri J, Kuroda K, Tokita S, Kuroda M, Takeda S (2010) Strategy for laparoscopic cervical myomectomy. J Minim Invasive Gynecol 17(3):301-305

4. Lasmar RB, Barrozo PR, Dias R, Oliveira MA (2005) Submucous myomas: a new presurgical classification to evaluate the viability of hysteroscopic surgical treatment-preliminary report. J Minim Invasive Gynecol 12(4):308-311

5. Lasmar RB, Lasmar BP, Celeste RK, da Rosa DB, Depes DB, Lopes RG (2012) A new system to classify submucous myomas: a Brazilian multicenter study. J Minim Invasive Gynecol 19(5):575580. doi:10.1016/j.jmig.2012.03.026

6. Di Spiezio SA, Mazzon I, Bramante S, Bettocchi S, Bifulco G, Guida M, Nappi C (2008) Hysteroscopic myomectomy: a comprehensive review of surgical techniques. Hum Reprod Update 14(2):101-119

7. Leone FPG, Calabrese S, Marciante C, Cetin I, Ferrazzi E (2012) Feasibility and long-term efficacy of hysteroscopic myomectomy for myomas with intramural development by the use of nonelectrical "cold" loops. Gynecol Surg 9:155-161

8. Lasmar RB, Barrozo PRM, da Rosa DB, Lasmar BP, Modotte WP, Dias R (2009) Hysteroscopic myomectomy in a submucous fibroid near from tubal ostia and $5 \mathrm{~mm}$ from the serosa: a case report from the Endoscopy Service of Ginendo-RJ. Gynecol Surg 6(3):283-286

9. Lasmar RB, Dias R, Barrozo PRM, da Rosa DB (2007) Hysteroscopic myomectomy in a submucous fibroid $3 \mathrm{~mm}$ from the serosa: a case report. Gynecol Sur 4:149-152

10. Chang WC, Chen SY, Huang SC, Chang DY, Chou LY, Sheu BC (2010) Strategy of cervical myomectomy under laparoscopy. Fertil Steril 94(7):2710-2715

11. Chu CM, Acholonu UC Jr, Chang-Jackson SC, Nezhat FR (2012) Leiomyoma recurrent at the cervical stump: report of two cases. J Minim Invasive Gynecol 19(1):131-133

12. Bettocchi S, Ceci O, Di Venere R, Pansini MV, Pellegrino A, Marello F, Nappi L (2002) Advanced operative office hysteroscopy without anaesthesia: analysis of 501 cases treated with a 5 Fr. bipolar electrode. Hum Reprod 17(9):2435-2438 